\title{
Frontières
}

\section{Petit traité du devenir rien à travers quelques figures féminines durassiennes}

\section{Marie-Soleil Roy}

Volume 21, numéro 2, printemps 2009

Détresse psychique et antidépresseurs

URI : https://id.erudit.org/iderudit/039456ar

DOI : https://doi.org/10.7202/039456ar

Aller au sommaire du numéro

Éditeur(s)

Université du Québec à Montréal

ISSN

1180-3479 (imprimé)

1916-0976 (numérique)

Découvrir la revue

Citer cet article

Roy, M.-S. (2009). Petit traité du devenir rien à travers quelques figures féminines durassiennes. Frontières, 21(2), 41-45.

https://doi.org/10.7202/039456ar
Résumé de l'article

Cet article se propose d'interroger trois oeuvres du corpus de Marguerite Duras, soit Le Vice-consul (1966), Détruire dit-elle (1969) et India Song (1973), en vue de souligner une étrange tension entre la faim, le désir et leur négation, à savoir l'entrée en anorexie. La question que cet article tente d'élucider est celle de la place du langage au centre même de la détresse et de ses liens avec la mise en récit du corps. C'est par le biais de l'analyse des corps maigres que nous aborderons un désir de perte qui, s'il répond à la nécessité d'enrayer la douleur, tend à l'abolition même des sujets en cette absence de toute mise en récit (qui s’apparenterait à une mise en corpus, une mise en corps). 


$\begin{array}{lllllll}R & T & \text { I } & \text { C } & \text { L } & \text { E } & S\end{array}$

\section{Résumé}

Cet article se propose d'interroger trois œuvres du corpus de Marguerite Duras, soit Le Vice-consul (1966), Détruire ditelle (1969) et India Song (1973), en vue de souligner une étrange tension entre la faim, le désir et leur négation, à savoir l'entrée en anorexie. La question que cet article tente d'élucider est celle de la place du langage au centre même de la détresse et de ses liens avec la mise en récit du corps. C'est par le biais de l'analyse des corps maigres que nous aborderons un désir de perte qui, s'il répond à la nécessité d'enrayer la douleur, tend à l'abolition même des sujets en cette absence de toute mise en récit (qui s'apparenterait à une mise en corpus, une mise en corps).

Mots clés: Marguerite Duras langage - oubli - poison - maigreur anorexie.

\section{Abstract}

This essay offers a reading of three works of Marguerite Duras: Le Vice-consul (1966), Détruire dit-elle (1969) and India Song (1973). It explores some "eating disorders" that are involved in the itinerary of three feminine protagonists. This essay will attempt to explore the role of the language in the very core of the distress and its links with the narrative construction of the bodies. This demonstration will be done by analysing the thin bodies that symbolise the subject's will to disappear in a place where no pain lies.

Keywords: Marguerite Duras language - forgetting - poison leanness - anorexia.

\title{
Petit traité
}

\section{du devenir rien}

\section{à travers quelques figures féminines durassiennes}

\author{
ET ALICE N'AVAIT JAMAIS OUBLIÉ \\ QUE SI L'ON BOIT UNE BONNE PARTIE DU CONTENU \\ D'UNE BOUTEILLE PORTANT L'ÉTIQUETTE : POISON, \\ ÇA NE MANQUE PRESQUE JAMAIS, TÔT OU TARD, \\ D'ÊTRE MAUVAIS POUR LA SANTÉ. \\ LEWIS CARROLL, ALICE AU PAYS DES MERVEILLES.
}

\section{Marie-Soleil Roy, \\ candidate au doctorat, Département des littératures de langue française, Université de Montréal.}

«Bois-moi » intime l'étiquette sur la bouteille que soulève Alice parachutée dans le vide, comme en un puits profond, du terrier d'un étrange lapin blanc. Comme il n'y est pas mention de poison, rassurée, la fillette ingurgite la boisson. Passeport direct pour le Monde des Merveilles, la boisson, si elle a raison de la patience de la jeune fille, lui ouvre des portes autrement plus troublantes: après avoir été réduite à la taille d'un lilliputien, elle devient géante, retrouvant avec peine sa taille normale. Son corps se transforme au gré des substances qu'elle consomme. Le corps se métamorphose, grandit, raccourcit, s'allonge, grossit, maigrit. L'assaut est donné par l'innocence même - simple breuvage, simple nourriture - à l'innocence incarnée par la petite fille à qui l'on a appris à se méfier des étiquettes où il était écrit poison.

Poison: n. m. est issu (v. 1100) du latin potionem, accusatif de potio, -onis «action de boire», d'où par métonymie «breuvage, boisson»; par spécialisation, le mot a désigné un breuvage médicinal (potion) et un breuvage empoisonné, un philtre magique (Rey, 2006, p. 2819).

Entre le boire et l'empoisonnement, une intimité à interroger, comme entre nourriture et métamorphose. Mais puisque tel poison ne se présente pas toujours ainsi, auréolé de son étiquette qui dit qui il est et ce qu'il cause, méfiance et prudence ne sont d'aucune utilité: Alice peut bien parler, peut savoir lire, elle reste devant l'énigme de la boisson entièrement démunie - elle doit boire pour savoir. Ainsi le 
poison s'insinue [-t-il] en elle: un démon comme un autre, celui de la curiosité, est né qui enjoint à décoder tous les signes sans égards pour la vie, pour sa sauvegarde. Alice entre dans l'interprétation des signes par le biais de son corps tout entier qui subit les assauts des substances ingurgitées, modifiant par le fait même, comme le fait remarquer Isabelle Meuret dans L'anorexie créatrice, "sa perception du monde» (Meuret, 2006, p. 62), l'obligeant à choisir, qui des mots, qui de la nourriture, la trahit et menace de la faire disparaître.

Si c'est toujours par le regard qu'elle est prise, la faim apparaît ainsi comme un moteur assez puissant pour lui faire traverser les espaces les plus étranges, mais aucun aliment ne peut lui donner la satisfaction escomptée: toute substance ingurgitée, quand elle réussit à mettre la main sur l'aliment convoité, la propulse hors d'elle-même, en des territoires de plus en plus étranges. Son regard lui ouvre le monde de la faim et, dans un renversement presque parfait, la faim lui dessille le regard. Le Pays des Merveilles est une aventure des signes qui ne laisse jamais sa voyageuse endormie, car aucun signe ne se dévoile tel qu'il prétend être. La voyageuse devient voyeuse et c'est un crime qui se paie par la tête. "Qu'on lui coupe la tête», scandent roi et reine afin que disparaisse le chat qui a osé, du regard, défier le roi.

Regard: déverbal de regarder [luimême] dérivé de garder qui avait la valeur d'«avoir l'œil sur, regarder», avec le préfixe re-indiquant à la fois le mouvement en arrière, en retour et la réitération (Rey, 2006, p. 3141).

Mais aucun garde ne peut s'emparer du chat qui s'évanouit de lui-même, alors «le Roi et le bourreau se mirent à courir comme des fous dans tous les sens pour [...] retrouver [la tête du chat] »(Carroll, 1961, p. 133 [notre soulignement]). Le sens de cette folie est de ne pas croire à ce qu'on ne peut voir. Du même coup, quelque chose d'autre émerge qui dit que la folie participe de l'impossibilité de faire tenir ensemble des sens disparates: le regard fou, c'est le regard hagard, égaré, agrandi par la peur, où pointe quelque chose de sauvage. $\mathrm{Ce}$ regard ne construit rien, ne sait comment rassembler en un phénomène explicable ce que ses sens, en un mouvement de pure synesthésie, perçoivent. C'est aussi le regard fixe qui embrasse tout et ne voit rien, ou ne voit qu'une seule chose, toujours la même, imperturbablement.

Mais comment et quoi et pourquoi rassembler? pourrait demander une autre voix, celle de Marguerite Duras que je veux interroger ici à travers quelques écrits - soit Le Vice-consul (1966), Détruire dit-elle (1969) et India Song (1973) - qui mettent littéralement en scène l'impossibilité de s'en remettre aux signes, au sens, pour tracer la voie d'un mieux-être. Car j'y retrouve, dans cette œuvre que j'atteins ici au détour d'un chemin sinueux auquel je reviendrai, une tentative de se soustraire à ces mouvements auxquels le corps d'Alice est soumis et qui sonnent "comme un adieu aux merveilles de l'enfance» (Meuret, 2006, p. 63) et une entrée dans le monde séparé, où mot et chose ne coïncident plus. Et où même toute traque étymologique ne peut être d'aucun secours...

\section{"NE FAISONS RIEN..., "}

Dans l'œuvre de Marguerite Duras, on a déjà pu l'évoquer, les regards se révèlent le plus souvent crevés, traversés de la brûlure du soleil, parfois fixes ou fixés comme on dirait immobiles, mais aussi attachés; le regard du vice-consul de Lahore paraît mort, c'est celui, pense-t-on, d'un autre. Ouverts, les yeux voient dans un regard vide, regardent le plus souvent le vide. Un autre monde captive certains personnages qui passent, errent ou s'endorment en des lieux de passage où jamais l'on ne reste. Cet autre monde est celui d'une douleur autour de laquelle on se meut, mais qu'on ne pointe jamais. C'est comme si aucune origine n'existait pour dire précisément la douleur. Il y a, chez Duras, cette histoire d'enfant mort-né - séparé à la naissance d'une mort séparée (Duras) -, il y a la guerre, il y a des amours qui furent malheureux ou trop heureux, mais ce n'est jamais tout à fait cela la douleur. Ce serait plutôt un état qui modélerait l'être et le ferait advenir comme en perpétuelle détresse.

Je me propose ici de suivre l'itinéraire de quelques figures féminines qui hantent littéralement les pages écrites de l'œuvre de Marguerite Duras: ces itinéraires convoquent l'idée selon laquelle l'absence de récits - de mises en récit autour d'événements traumatiques ouvrent sur d'étranges libérations paradoxalement mortifères. Cette absence de mise en récit, cette impossibilité où le sujet est d'être dit se déplace sur un autre plan: le corps, en sa maigritude, apparaît comme le témoin privilégié d'un mal-être qui, faute d'être écrit ou dit, propulse le sujet dans un non-être irréversible, là où chose et mot pourraient coïncider comme en un lieu où le Gros Coco (autrement connu sous le nom de Humpty Dumpty) que rencontre Alice sur son chemin pourrait enfin affirmer qu'il a un nom référant à sa propre forme.

Duras aurait confié à Enrique VilaMatas, qui fut son locataire dans les années 1970 - et à d'autres peut-être -, qu'elle écrivait pour ne pas se suicider (VilaMatas, 2006, p. 30). C'est là une chose qui apparaît dans Écrire (1993), car dans la solitude dans laquelle on peut la penser recluse, dans la solitude dans laquelle certains de ses personnages semblent enfermés, exilés, c'est, comme elle l'écrit alors, «Ou la mort, ou le livre. Mais avant tout ça veut dire l'alcool. Whisky, ça veut dire» (Duras, 1993, p. 19). Je commencerai par la fin de cette citation, par là où il y a justement absence de faim, que le seul désir de se perdre dans et par une boisson, un poison peut-être, peut-être un philtre magique, quelque chose qui fasse oublier, sans que cet oubli n'ait un objet - un oublier intransitif.

Oublier: v. tr., qui succède aux formes oblier (v. 980) et ublier, est issu d'un latin populaire ${ }^{\circ}$ oblitare, réfection, sur le participe passé oblitus, du verbe classique oblivisci «ne plus penser à qqc., perdre de vue». C'est un mot de la même famille que oblinere, « couvrir d'un enduit », d'où « raturer, effacer», qui contient une racine indoeuropéenne ${ }^{\circ} l e i$ - signifiant «verser un produit gras» et qui est représentée, avec un élargissement en ${ }^{\circ} w$, dans le latin levis «poli, lisse» (non roman) et le grec de même sens leios, peut-être également dans le latin lima (lime). (Rey, 2006, p. 2504)

L'oubli en passerait par un recouvrement, un voilement ou plutôt une sorte de polissage, comme s'il en allait d'une réduction: enlever ce qui est de trop. C'est aussi le refus, ou l'impossibilité, de garder. Parle-t-on du regard d'Anne-Marie Stretter (personnage omniprésent dans l'œuvre de Duras) ? C'est «féroce» ou «doux» qui vient, c'est-à-dire rien. L'un annule l'autre, et inversement. Ainsi, raturant, rayant, par le mot et son contraire, l'écriture durassienne rend certains personnages insaisissables.

Si c'est à la mendiante du Vice-consul (1966) qu'on a pu associer ce désir de se perdre, il appert dans India Song (1973) qu'«elle aussi »a «toujours cherché à se perdre, en somme, depuis le commencement de sa vie»(Duras, 1973, p. 135). «Elle aussi », c'est Anne-Marie Stretter, la femme de l'ambassadeur de France aux Indes. De ce désir de se perdre, il aurait résulté un nomadisme sans fin, de la France à Venise, en passant par toutes les capitales du monde asiatique, pour finir à Calcutta, dans les eaux du Gange, là où la mendiante chauve chasse sa nourriture.

Si le mari d'Anne-Marie Stretter a déjà pu témoigner, à travers quelques poésies, de son désir d'écrire, sa femme l'en a vite dissuadé, lui affirmant qu'il ne fallait pas écrire, plutôt ne rien faire: «[i]l ne faut pas écrire, restons ici, de ce côté-ci, en 
Chine, aux Indes, de la poésie personne ne sait, il y a dix poètes sur des milliards d'hommes chaque siècle... Ne faisons rien, restons là... rien... » (Duras, 1966, p. 136). Elle demande d'appartenir au commun des mortels, de se fondre dans ce qui n'a pas de voix par le fait d'être enterré dans la multitude (des milliards d'hommes), de se fondre dans l'anonymat faute de ne pouvoir avoir la voix qu'il faudrait pour traverser la surdité des hommes, leur nonsavoir devant la poésie. Devant la menace de remous que l'écriture pourrait créer, la femme de l'ambassadeur affirme clairement son désir de repos, son désir de rien («ne faisons rien, restons là... rien») qui l'apparente, en sa maigreur, à l'anorexique $^{1}$. Elle dit aussi en creux la folie, le non-sens de la présence blanche dans les pays asiatiques, l'absurdité des colonies avec le désir, pourtant, de se fondre, de se couler dans cette absurdité. Mais le désir qui sous-tend - si tant est qu'on puisse parler de désir - ces paroles ne serait-il pas de l'ordre d'un appel au néant? Après ces paroles rapportées, l'épouse de l'ambassadeur boit du champagne, boit du whisky, boit des nuits entières accompagnée de ses jeunes amants anglais...

Le nomadisme, qu'il soit spatial ou sexuel chez Anne-Marie Stretter, s'accompagne d'un détachement progressif de ce qui pourrait sauver de l'horreur: elle semble plutôt vouloir s'y enliser, s'y perdre, passer la frontière, franchir l'étroitesse de l'espace où elle se garde de la famine de Calcutta. Si l'ambassade où elle donne ses soirées à une élite blanche s'entoure de clôtures qui protègent des lépreux et des mendiants, cette frontière n'est jamais réellement perméable: passent au travers les cris des lépreux, le chant de la mendiante, quand les grilles ne sont pas littéralement ouvertes afin de donner restes de nourriture et eau aux affamés de Calcutta. Mais quelque chose d'une certaine invisibilité accompagne Anne-Marie Stretter: de blanc ou de noir vêtue, elle intrigue, attire, fait qu'on parle d'elle, qu'on cherche à savoir. Elle appartient étrangement au plus clair et au plus sombre, faisant que les récits autour d'elle ne peuvent que se transformer en on-dit ${ }^{2}$. Parce qu'elle intrigue, elle fait naître autour d'elle une série d'interrogations: "Que dissimule cette ombre qui accompagne la lumière dans laquelle apparaît toujours AnneMarie Stretter?»(Duras, 1966, p. 109.) Aucun récit ne peut saisir cette femme qui apparaît et donc, menace à l'instar du fantôme, de disparaître: elle est à la fois l'ombre et la lumière, le blanc et le noir, à la fois vieille et enfantine, belle et laide. Elle serait le mouvement insaisissable, et la dire consisterait à la fixer, à la figer et, donc, à ne pas la dire.

Mais cette impossibilité d'être mise en récit, cette résistance à l'assaut du sens qui voudrait façonner de l'identité révèle autre chose. Afin d'éclairer ce personnage d'Anne-Marie Stretter - femme qui donne envie de vivre, femme qui rend fou d'espoir, femme accueillante "vers qui vont toutes les vagues et toutes les douleurs » (Duras, 1966, p. 120) -, il m'apparaît intéressant d'en passer par les personnages féminins de Détruire dit-elle, récit de Duras paru en 1969, soit entre Le Vice-consul et India Song.

\section{MOURIR DE GUÉRIR DE L'ENFANT MORT-NÉ}

C'est dans un hôtel bien souvent que se rencontrent les personnages de Duras. Là, des regards sont échangés, voire donnés sans retour, bien plus que des paroles qui le plus souvent font place au silence - silence qui scande la plupart des récits, comme une sorte de didascalie obligeant le lecteur à prendre le pouls des non-dits qui se cachent dans le si peu qui est si péniblement dit.

C'est dans un hôtel que les regards multiples de Détruire dit-elle se chargent de questions. Dans ce récit publié aux Éditions de Minuit, en 1969, il y a d'abord elle, qui n'est pas le sujet du titre, et qu'on saura plus tard s'appeler Élisabeth Alione, venue seule, dont la mémoire, celle des événements immédiats, semble osciller: elle voit, regarde, mais ignore être regardée; puis il y a devant elle, mais échappant à son regard, sur la table de la terrasse où elle s'est assise près des tennis, un livre et des flacons de pilules. Elle ne lit donc pas, elle tente, mais repose toujours le livre. À côté du livre, la narration indique qu'il y a des "flacons de pilules blanches» qu'on saura plus tard être des calmants, que la femme prend avec de plus en plus de légèreté, bien que la peur éprouvée ne se fasse plus profonde, plus insistante. Et puis, sur les autres tables de cet hôtel, que l'étymologie rattache à l'hôpital, on peut imaginer, tel que l'incipit le propose, «d'autres flacons, d'autres livres» (Duras, 1969, p. 10). "Sur d'autres tables, d'autres flacons, d'autres livres » : à travers ce parallélisme, les livres et les flacons se présentent sur un pied d'égalité. Livres et flacons constitueraient le remède du solitaire mis en quarantaine en cet hôtel par des proches qui redoutent la faiblesse des êtres qu'ils confient à ce lieu de passage où personne ne reste vraiment, où personne n'est vraiment là, flottant dans une sorte d'anonymat tranquille et vaporeux que seuls deux Juifs - c'est ainsi qu'ils sont présentés au lecteur -, soit Stein et
Max Thor, tentent de pénétrer. L'un d'eux - mais lequel? - est écrivain. Il tente de puiser à même la maladie, voire la folie, répandue en cet hôtel qui lui fut "conseillé par un collègue universitaire», une inspiration, quoique le terme plus médical de sujet apparaîtra plus juste.

Devant elle, il y a le livre. Commencé

depuis son arrivée à lui ? ou encore avant?

Près du livre il y a deux flacons de pilules blanches. Elle en prend à chaque repas. Quelquefois elle ouvre le livre. Puis elle le referme aussitôt. Elle regarde le tennis.

Sur d'autres tables d'autres flacons, d'autres livres (Duras, 1969, p. 9-10).

Et si j'entends le parallélisme jusqu'au bout, c'est dire que les flacons ou médicaments servent au même titre que les livres, dont c'est sans doute là le but premier, de divertissement afin de détourner de la douleur. Détourner de la douleur : livres et pilules y contribuent.... mais à quel degré? Le livre n'est jamais lu. Ouvert, il est vite refermé - comme si la vraie vie n'y était pas ; on saura plus tard, grâce à l'œil inquisiteur de Stein qu'il s'agit d'un roman de train, c'est-à-dire «rien ». Personne, en fait, ne lit vraiment. Personne, en fait, n'écrit non plus en ce lieu étrange que non seulement l'étymologie, mais surtout la population qu'il y enferme, assimile à une sorte d'hospice ou d'hôpital où l'on ne reste pas, bien que certains y reviennent chaque année à l'été. Dans cet hôtel que borde une forêt classée "monument historique ${ }^{3}$ » (Duras, 1969, p. 126), le jour est donné aux siestes. On fait presque silence sur la nuit, lieu de l'amour et de l'écrit, vite donnée à lire comme lieu paradoxal de l'amour impossible et de l'impossibilité d'écrire.

En effet, la nuit se constitue comme le lieu privilégié de l'écriture pour Max Thor, époux de la jeune Alissa et vieux professeur d'université qui dira enseigner «l'histoire de l'avenir ». Mais l'histoire même s'élève contre toute tentative de décrire, ou d'écrire: "chaque nuit, depuis que je suis arrivé dans cet hôtel, je suis sur le point de commencer... je n'écris pas, je n'écrirai jamais... oui, chaque nuit change ce que j'écrirais si j'écrivais » (Duras, 1969, p. 38-39). Ici, quatre fois le verbe «écrire» conjugué à la première personne du singulier, soit le «je» de Max Thor, mais quatre fois différentes, à des temps qui obligent à questionner la temporalité dans laquelle le sujet se déploie: un présent de l'indicatif accompagné d'une négation, un futur tout aussi rayé par un «jamais ». Un conditionnel suivi d'un imparfait posant l'hypothèse: si j'écrivais, chaque nuit transformerait le sujet de l'écriture. Chaque nuit obligerait à rayer, à recommencer, à raturer, à ne 
jamais pouvoir écrire; le mouvement de l'oubli évoqué plus haut fonctionne de la même manière: le mouvement de la rature sur l'inscription. Ainsi n'y aura-t-il jamais d'écriture possible; jamais de commencement. Le commencement, tout commencement se révèle ici, comme ailleurs dans l'œuvre, frappé d'impossibilité et pourtant appelé. Comme si tout commencement était illégitime à n'être pas constamment répété, réécrit.

Le problème se situe précisément dans cette question: comment commencer sans crainte de tout figer et de perdre ce faisant ce qui est la vie même? Comment sortir de la peur de commencer? Comment trouver un commencement qui ne fige pas tout dans un éternel présent? En somme, comment commencer, ou recommencer, quand se profile surtout la figure absente de l'enfant mort-né - de ce qui était promesse, mais vite devenu une absence qui oblige à «tout imaginer ${ }^{4}$ ? Comment commencer autrement que par l'accident, par ce qui était inimaginable, n'était pas attendu? Faire entrer l'accident comme valeur de commencement implique une redéfinition de la vie que les personnages ne sont pas tous prêts à endosser, d'où la nécessité du titre qui implique une destruction.

Les personnages empêchés, soit de parler, soit d'écrire, ou encore empêchés dans leur mouvement - la plupart de ceux qu'on appelle les «malades » de l'hôtel regardent la forêt, mais personne n'y pénètre, elle représente un danger, un inconnu, la possibilité d'un accident - sont nombreux dans ce récit, nombreux à dire non. Leur mouvement, celui d'Élisabeth Alione par exemple, devient peu à peu presque rien : ils forment des pierres qui s'endorment au soleil, des longs corps immobiles, les yeux grand ouverts sur le sommeil, où l'immobilité, voire la pétrification, est pressentie comme protection contre le dehors. De fait, la forêt qui borde l'hôtel apparaît hostile à Élisabeth Alione, dangereuse : elle la voit, la regarde, mais jamais elle ne voudra y entrer. La seule qui voudra s'y aventurer, c'est l'autre femme, Alissa, l'épouse de Max Thor, celle qu'on a dit folle et qui n'a peur de rien, qui se fait accueil, hôte hospitalière, qui ne sait aucune limite et qui va dans la forêt pour l'amour, se donner à Stein, son amant. Entre Élisa et Alissa va se mettre en place un étrange phénomène d'écho où les deux femmes - la première ayant perdu une petite fille en couches, mais faisant obstinément silence sur cet «accident» et la seconde d'à peine 18 ans étant considérée comme "déjà un enfant» (étrange formulation qui semble dire que l'avenir appartient aux enfants) -, rassemblées autour d'un miroir vont finir par se ressembler: « la même taille, la même peau, la même couleur de peau, les mêmes cheveux» (Duras, 1969, p. 100-101), etc. Cette dualité répète étrangement les deux motifs de l'eau et de la pierre, de la liberté et de la captivité qui se partagent l'œuvre: l'une serait coulante, fluide, aventurière, libre, l'autre, tout en silence, en sommeil, captive des murs de l'hôtel, captive des calmants qui l'endorment, d'un mari dont elle dit faire «ce qu'il veut»(Duras, 1969, p. 62), captive de la culpabilité entourant l'événement de l'enfant mort-né qu'elle a peur que son mari lui reproche, captive de sa propre vérité, car elle cache vraisemblablement quelque chose sous son regard vide et son faux sommeil.

Élisabeth Alione, née Élisabeth Villeneuve, serait devenue, par les liens du mariage à l'âge de 18 ans, une sorte d'étrangère à elle-même: Alione ou aliénée qui ne semble penser qu'à travers son mari absent. Sauf quand elle dit la peur éprouvée par l'hostilité du dehors, il n'y a pas de je qui tienne. En fait, peu de paroles et une solitude que marque aussi son nom anglicisé, amputé du «i » de l'italien: alone. Il s'agira d'une femme seule depuis le mariage, d'une femme laissée à elle-même, le travail obligeant son mari à s'absenter souvent.

Aliénée, liée, et dont l'histoire reste énigmatique autour de sa décision à aller «faire le deuil» de l'enfant dans un hôtel bordé d'une forêt, Élisabeth Alione fascine par son appartenance au monde de l'invisible, fermée qu'elle est sur une mort d'enfant qui l'a fatiguée, épuisée, dépossédée. Aliénée jusque dans sa parole, comme lorsqu'elle affirme à une Alissa interrogatrice que «le docteur m'a dit d'éviter d'en parler»(Duras, 1969, p. 64). Aliénée jusque dans ses désirs quand Alissa lui réplique qu'elle pourrait faire à sa tête, n'écouter personne et qu'elle répond: «je n'en ai pas envie» (Duras, 1969, p. 65). Pour l'heure, elle est dans le sommeil et dans la peur de la forêt où Alissa l'invite à pénétrer. Car la forêt, c'est l'inconnu, le mystère, le danger. On a pu l'associer à l'inconscient: on pourrait y voir le lieu possible de la réalisation des désirs. Mais le sommeil d'Élisabeth apparaît sans rêves, un no man's land, un néant assuré par les médicaments et l'interdiction, donnée par le médecin, de dire quoi que ce soit sur l'événement de l'enfant mort-né - tentant d'assurer à sa patiente, par delà le silence, que la mort sombre dans le nonévénement ${ }^{5}$.

La forêt se présente donc comme une frontière visible, à ne pas franchir, qui rend possible le repos: les murs d'une prison, que l'autre personnage féminin n'aura de cesse de traverser. Ainsi, deux positions se dessinent: d'un côté, un dormir, si proche de la mort ( Elle dort. Elle est grande, ainsi morte, légèrement cassée à la charnière des reins. Elle est mince, maigre» [Duras, 1969, p. 11]) qui pense la guérison comme endormissement du mal, engourdissement des désirs et le désir d'un anéantissement qui est loin d'un ensommeillement, de l'autre, le désir de détruire qui hante le personnage enfantin, fou, d'Alissa dont le nom se fait un écho lointain à l'Alice de Carroll. Il y a d'un côté Élisa, de l'autre Alissa. Un miroir se dresse entre les deux qui les sépare et les rassemble autour d'un désir d'abolition.

\section{DE L'AUTRE CÔTÉ DU MIROIR, IL N'Y A PAS D'HISTOIRES}

Il est vrai que maintenant on ne raconte plus rien dans les romans... C'est pour ça que j'en lis si peu... que... Marguerite Duras, Détruire dit-elle.

Un livre sans images ni conversation propulse Alice de l'autre côté. Mais qu'y a-t-il de l'autre côté? Là où elle lit - au contraire du personnage de Détruire ditelle qui ne lit plus rien -, Anne-Marie Stretter n'est pas visible. On sait qu'elle est occupée par quelque chose. On croit qu'elle lit, car «que ferait-elle d'autre, chez elle, enfermée? Des colis de livres arriv[ant] de France à son nom » (Duras, 1966, p. 94). On imagine alors, on ne fait qu'imaginer la position qui pourrait être la sienne lorsqu'elle se retire pour lire, seule, « dans un coin caché de sa résidence, dans un office abandonné, recroquevillée sur elle-même dans une pose extravagante» (Duras, 1966, p. 108). Du livre, on ne voit rien, on n'imagine rien. Il n'y a pas d'objet à la lecture: il s'agit d'une occupation qui se referme sur le vide, qui se referme sur du corps, sur la position d'un corps. Lire évoquera donc la mort, et l'imaginer, cette femme, reviendra toujours à ne rien savoir: de l'autre côté des murs où elle se réfugie, aucune histoire ne tient, aucune histoire n'existe. D'ailleurs, en sa maigreur, elle fait qu'on se questionne: sa maigreur témoignerait de quelque chose, mais ce quelque chose reste inexpliqué. On lui suppose une histoire:

Que s'est-il passé à la fin de la première année de son séjour? Cette disparition que personne n'expliquait? Une ambulance au petit jour a été vue devant la résidence. Tentative de suicide? Ce séjour dans les Montagnes du Népal est resté inexpliqué. Cette maigreur à son retour fait peur.

Pas d'autres différences? Elle reste maigre, c'est tout. On dit que c'est à cause d'un amour ou malheureux ou trop heureux avec Michael Richard. (Duras, 1966, p. 110) 
«Elle reste maigre, c'est tout»: cette observation fait écho au «ne rien faire» évoqué plus haut, et permet de tisser un lien entre le corps et la parole, tous deux en quelque sorte niés en ce qu'ils sont voués à la disparition. En regard de cette disparition, il vaut mieux ne rien faire, ne pas écrire puisque tous les signes reviennent au même. Regarder ou lire Anne-Marie Stretter, c'est se tenir devant un mystère, une non-histoire: à la fois belle et laide, vieille et enfant, légère et de pierre en sa posture de gisante lorsqu'elle dort, vivante et déjà morte, c'est une femme, comme dira le Vice-consul, qui n'a pas de préférence: elle est à qui veut d'elle. «Dormeuse debout», elle se déplace en silence, sans parole, sans savoir...

\section{UN DEVENIR ENFANT}

Mon Dieu, accordez-moi

de devenir rien

Simone Weil, Cahiers VI

L'enfant, l'infans : «n.m. est la francisation (en-) $\left(\mathrm{XI}^{\mathrm{e}} \mathrm{s}\right.$.) de l'emprunt très ancien infans (fin $\mathrm{X}^{\mathrm{e}} \mathrm{s}$.) au latin classique infans, infantis, à l'accusatif infantem: le mot signifie proprement "qui ne parle pas" » (Rey, 2006, p. 1239). Aucune parole ne sera émise. Anne-Marie Stretter dort, rajeunie, assise "dans une pose enfantine, disloquée», puis, tranquillement, elle devient «plate, légère», prend la « rectitude simple d'une morte» (Duras, 1966, p. 197). Son corps mime, en son sommeil, un retour à l'informe, à l'infans. Parle-t-elle? Elle répète. Dort-elle? Elle régresse. Tout est devenu immobile. Anne-Marie Stretter est une «eau qui dort»: elle fascine, on s'y abîme. Récit lisse sur lequel personne n'a prise, elle est la grâce au milieu de laquelle l'horreur sévit, se tenant, écrit Duras (1973) à la toute fin de India Song, "au milieu d'elle [de l'horreur] avec une grâce où tout s'abîme, dans un inépuisable silence».

Si l'on assiste effectivement à une métamorphose du personnage d'AnneMarie Stretter à travers l'écriture qui la fait passer de femme à enfant à morte, il va de pair avec le mouvement voyageur qui fut le sien, passant de l'Occident à l'Orient, vers l'inconnu, l'étrangeté, vers un lieu où il n'est «ni pénible ni agréable de vivre», «ni facile ni difficile» (Duras, 1966, p. 109), où ce n'est rien. De même l'Alissa de Duras qui résonne à nos oreilles un peu carrollienne est « déjà » une enfant, toute faite d'un désir indomptable, celui, entre autres, de détruire, de n'être d'aucun commencement, d'être sans tête, comme folle, perméable à tout, poreuse, éprouvant ce que l'autre éprouve. Riant à travers son sommeil final qui lui fait une tête de morte, elle se fait enfant, oscillant entre le rêve et la réalité; proférant quelques paroles prophétiques, elle se fait pythie lisant la destruction à venir, comme si de certains voyages on ne pouvait revenir tout à fait sans que parole et corps ne s'en trouvent, d'une quelconque manière, atteints.

Ainsi, les lieux nous font et nous défont: il y a cette pensée chez Duras qu'il faut garder en mémoire. En ce sens, l'horreur de Calcutta est un poison que boit jusqu'à la lie Anne-Marie Stretter, et la plupart des personnages durassiens qui ont peine à reconnaître leurs limites dans une sorte d'illimité où ils finissent par disparaître, tout en s'abreuvant de la détresse des autres, en s'en laissant pénétrer. Si le mot détresse provient d'abord de la destresse qui signifie "chose étroite, étroitesse", l'étroitesse, à la fois du lieu colonial comme espace de divisions ethniques, à la fois de l'esprit de la communauté blanche des colonies, s'inscrit dans le corps frêle, long et maigre, d'Anne-Marie Stretter, dans son discours proche du silence, dans l'immobilité de ses endormissements. Ainsi la détresse aura été matée par le vide, par le rien. Et par l'impossibilité de résister au lieu, par la nécessité d'adhérer, de ne faire plus qu'un avec lui, ou encore avec l'autre. Alors c'est la mort, écrivait Duras, que seul le livre, tel un philtre, peut suspendre. Mais il s'agira toujours d'une potion : bois-moi, lis-moi, mange-moi, dors-moi... Étiquette sans aucune mention de poison. Est-ce pour autant sans effet?

\section{Bibliographie}

CARROLL, LEWIS (1961). Alice au pays des merveilles. De l'autre côté du miroir, trad. de Jacques Papy, Paris, Gallimard, coll. «Folio classique».

DELVAUX, MARTINE (2005). Histoires de fantômes. Spectralité et témoignage dans les récits de femmes contemporains, Montréal, Presses de l'Université de Montréal, coll. «Espace littéraire».

DURAS, MARGUERITE (1966). Le Viceconsul, Paris, Gallimard, coll. "L'imaginaire».

DURAS, MARGUERITE (1969). Détruire dit-elle, Paris, Les Éditions de Minuit.

DURAS, MARGUERITE (1973). India Song, Paris, Gallimard, coll. "L'imaginaire».

DURAS, MARGUERITE (1993). Écrire, Paris, Gallimard, coll. «Folio».

ERNAUX, ANNIE (2001). L'événement, Paris, Gallimard, coll. «Folio».

MEURET, ISABELLE (2006). L'anorexie créatrice, Paris, Klincksiek, coll. "50 questions».

REY, ALAIN (dir.) (2006). Dictionnaire historique de la langue française, Paris, Le Robert.

VILA-MATAS, ENRIQUE (2006). «L'écrit et la mouche», Le Magazine littéraire, $\mathrm{n}^{\circ} 452$, avril.

\section{Notes}

1. L'anorexie, c'est étymologiquement, par le $a$ privatif, l'absence de désir.

2. Il serait d'ailleurs intéressant de répertorier le nombre de fois où apparaît cette expression dans Le Vice-consul et India Song: "On dit que... », faisant du mystère une chose qu'on ne peut jamais percer entièrement. Le flot de rumeurs que représentent les voix d'India Song prolonge le mystère: il n'y aura en définitive plus de récit pour dire Anne-Marie Stretter, mais des bribes de on-dit.

3. C'est bien ce que dit le roman : étrangement, il ne s'agit pas de classer la forêt «patrimoine mondial de l'humanité », mais de la considérer comme un «monument»...

4. C'est ce que Duras écrira, dans Les Cahiers de la guerre, à propos de la perte de son enfant, mort-né, et qu'on lui a enlevé avant qu'elle n'ait pu le voir, le prendre dans ses bras, obligée de tout imaginer...

5. Voir autour de l'événement que constitue l'avortement ou la venue d'un enfant mortné L'Événement d'Annie Ernaux (2001) et l'étude qu'en propose Martine Delvaux dans ses Histoires de fantômes (2005, p. 115-136). 